\title{
FORUM
}

\section{Longitude without Time}

\author{
J. J. Evans
}

The problem of determining longitude without reference to a timepiece is one which even today commands the interest of a number of mathematicians, astronomers and navigators. Having recently undertaken a crossing of the Atlantic in a small boat, I was particularly interested in Mr. Chichester's article and Mr. Sadler's comments in the January edition of this Journal.

The use of the visible horizon as a reference point for measuring the lunar distance from a celestial body is described in some of the older books on Nautical Astronomy. One method consists of observing the altitudes of the Moon and second body on opposite sides of the meridian and thence computing their respective hour angles. The sum of their hour angles is then equivalent to the difference in right ascensions of the two bodies. With this information, the lunar distance can readily be found and compared with tabulated or calculated lunar distances for given instants of G.M.T. to obtain the G.M.T. of observation. Mr. Chichester's variation of the method is certainly ingenious, and the need to compare lunar distances is thereby dispensed with. I cannot, however, see any reason why intervals as large as one hour between plots should be necessary: the overall size of the plot could well be reduced to one-sixth of the size, without loss of accuracy, by using times say $5 \mathrm{~min}$. on each side of the 'guessed at' G.M.T.

The actual method that Mr. Chichester employs to obtain his two 'fixes' presumably consists of making four sight reductions, two for one assumed G.M.T. and two for a second G.M.T., I hr. later or earlier, so that these times embrace the correct G.M.T. This being so, the assumed longitude would need to be adjusted by $15^{\circ}$ from the first to the second assumed G.M.T.s to maintain reasonably correct hour angles and to avoid unduly large intercepts.

Mr. Sadler approaches the plot in a somewhat different manner by calculating the position lines of Sun and Moon for time $T_{1}$ and then translating the position lines $15^{\circ}$ to the west to correspond to a time $T_{1}+\mathrm{I}^{\mathrm{h}}$ where the 'guessed at' G.M.T. lies approximately at the mean of the two times. A correction, for change in right ascension and declination in the corresponding time interval, is made to the Moon position line for time $T_{1}+\mathrm{I}^{\mathrm{h}}$. Mr. Sadler has pointed out that the Moon position line should be moved bodily towards or away from the Moon (depending upon whether it lies east or west of the observer's meridian) through a distance of $d \cos C_{ \pm}\left(4_{1}: 0-v\right) \cos \delta \sin C$, where $\delta$ is the declination, and $C$ is the parallactic angle. This corrects his previous statement that the Moon position line is translated through a small distance $P$ in position angle $P$, where $p \sin P=41^{\prime} \circ-v$ and $p \cos P=d$.

Clearly, for the best results using Mr. Chichester's method, the rate of change of altitude of the two bodies should be large. For maximum rate of change, latitude should be $0^{\circ}$ and azimuth $90^{\circ}$, in which case declinations would also be 
$0^{\circ}$. The implication is that in high latitudes and/or conditions when either of the bodies is near the meridian, poor results are likely.

It is interesting perhaps to note that under certain circumstances when the parallactic angle is small, implying that the Moon is near the meridian, an error in time may cause no relative shift of the Moon position line with respect to that of the Sun. An example to illustrate this is taken for G.M.T.s $10^{\text {h }}$ and $10^{\mathrm{h}} 15^{\mathrm{m}}$ on 16 June 1966 , Lat. $41^{\circ} \mathrm{N}$., $T$ altitude of Moon $65^{\circ} 4^{\prime}$. Calculation shows that longitude corresponding to $10^{\mathrm{h}}$ is $13^{\circ} 33^{\prime} \cdot 9 \mathrm{~W}$. and to $10^{\mathrm{h}} 15^{\mathrm{m}}$, $17^{\circ} 18: 9 \mathrm{~W}$., a difference of $3^{\circ} 45^{\prime}$ or $15 \mathrm{~min}$. in time. The Moon is east of the meridian, E.H.A. $11^{\circ} 15^{\prime}$ (approx.), T. Az. $1534^{\circ}$. The method is therefore of no use at all in this particular case.

In practice, I feel that an accurate latitude would be very difficult to determine, and in order to eliminate errors due to errors in latitude both bodies should lie on or near the same vertical circle, and preferably the prime vertical. Also, in order to reduce the effect of constant observational errors, both bodies should be observed on the same side of the meridian. In cases however where both bodies lie near the same vertical circle, Mr. Chichester's method falls down, owing to the difficulty of crossing the two position lines. This difficulty can be overcome, and plotting errors eliminated also, by treating the problem in a different way.

Due to the Moon's rapid change in right ascension, an error of $\mathrm{I} \mathrm{hr}$. fast on G.M.T. shifts the Moon position line to the east relative to the Sun position line by an amount $A$, where

$$
A= \pm r+\left(41^{\prime}: 0-v\right)
$$

and $r=15^{\circ} \sim$ change of Sun's hour angle in the corresponding hour, although for practical purposes $r$ can be ignored. At the same time, due to the Moon's change in declination, the Moon position line is shifted to the east or west relative to the Sun position line by an amount $B$, where

$$
B=d \sec \delta \cot C
$$

and the symbols have the same significance as previously indicated. If the Moon is east of the meridian and declination is increasing (Lat. and Dec. same names and $\mathrm{C}<90^{\circ}$ ), Moon position line will be shifted to the west. Conversely, Moon position line will be shifted to the east if Moon is west of the meridian or if Lat. and Dec. are of different names. Thus if $B$ is assigned a positive sign when the position line is shifted to the east, and a negative sign when shifted to the west, the separation of the two position lines in minutes of longitude for an error of I hr. fast on G.M.T. is given by the algebraic sum of $A$ and $B$. These two factors can be used to determine G.M.T. $(A+B \neq 0)$ by the longitude method of sight reduction. A G.M.T., which need not be very close to the correct G.M.T., is assumed and longitudes calculated for the Sun and Moon observations. If the assumed G.M.T. is correct these longitudes will coincide; if not, the error $t$ in minutes is given by

$$
(A+B) t / 60=K
$$

where $K$ is the separation of the calculated longitudes. The error is fast on G.M.T. if the Moon longitude lies to the east of the Sun longitude, and slow on G.M.T. if to the west. Factors $A$ and $B$ can readily be found, and $t$ thereby calculated. An example is given below. 
On 16 June 1966 at assumed G.M.T. $10^{\mathrm{h}} 5^{\mathrm{m}} \mathrm{o}^{\mathrm{s}}$ in latitude $41^{\circ} \mathrm{N}$., the true zenith distances of the Moon and Sun from observation are respectively $53^{\circ} 37^{\prime}: 8$ and $75^{\circ} 01: 0$ both east of the meridian.

By calculation, the longitude by Moon observation is $61^{\circ}{ }_{13}: 3 \mathrm{~W}$. and by Sun observation $6 I^{\circ} I_{5} \cdot 0 \mathrm{~W}$, , a difference of $I^{\prime} 7$, and it is apparent that the assumed G.M.T. is fast.

From the Nautical Almanac

$$
\begin{aligned}
A & =(-0 ! 2+41: 0-10 ! 6) \\
& =30 ! 2
\end{aligned}
$$

By the use of $A B C$ or other azimuth tables, the parallactic angle $C$ is found to be $5^{2} \cdot 6^{\circ}$. Whence,

$$
\begin{aligned}
B & =\left(-11 \sec 18^{\circ} 46^{\prime} 7 \cot 5^{\circ} 3^{\circ} 6^{\prime}\right) \\
& =-8: 9
\end{aligned}
$$

A realistic value of $B(-9 \cdot 0)$ is found using longitude correction tables based on the same formula.

Hence,

$$
(30: 2-8: 9) t / 60=1 \cdot 7
$$

and

so that

$$
t=4 \cdot 8 \mathrm{~min} \text {. (nearly) }
$$

$$
\begin{aligned}
\text { G.M.T. } & =10^{\mathrm{h}} 5^{\mathrm{m}} 0^{\mathrm{s}}-4^{\mathrm{m}} 4^{8^{\mathrm{s}}} \\
& =10^{\mathrm{h}} 0^{\mathrm{m}} \quad 12^{\mathrm{s}}
\end{aligned}
$$

Azimuths are not required in the computation.

One (perhaps dubious) advantage of this over the conventional lunar distance method is that the Moon and second body may be observed without loss of accuracy when on or near the same (but remote from the observer's) meridian.

\title{
Visual Indication of Direction for Ships
}

\author{
Commander G. V. Parmiter, R.N. \\ (Harbour Master, Port of London)
}

I was very interested in the recent correspondence regarding a direction indicator to be fitted to ships and while I note the experiments now being carried out by our Dutch friends there are several points which must be borne in mind.

In the first place, whilst sophisticated arrangements like illuminated arrows may be very satisfactory from the operational point of view, this sort of apparatus is just one more piece of expensive equipment that the shipowner must provide and $I$ foresee reluctance on his part if it is intended that this should come into general use. 